\title{
Pigmented corneal ring associated with orthokeratology in Caucasians: case reports
}

Jose M González-Méijome* OD PhD FIACLE FAAO

Javier González-Pérez ${ }^{\dagger}$ OD PhD FIACLE

Nery Garcia-Porta ${ }^{\dagger}$ OD MSc

Alberto Diaz-Rey* MD PhD

Manuel A Parafita-Mato ${ }^{\dagger}$ MD PhD

* Clinical and Experimental Optometry Research Laboratory (CEORLab), Center of Physics, University of Minho, Braga, Portugal

${ }^{\dagger}$ Ocular Surface and Contact Lens Research Laboratory, School of Optometry, University of Santiago de Compostela, Spain.

E-mail: jgmeijome@fisica.uminho.pt

Submitted: 24 August 2011

Revised: 4 March 2012

Accepted for publication: 5 March 2012
Background: The aim was to report the appearance of a pigmented ring in both eyes of two patients undergoing overnight orthokeratology.

Methods: Two Caucasian patients, one male and one female, were fitted with orthokeratology lenses to correct myopia between -2.00 and -2.50 DS with Paragon corneal refractive therapy lenses worn overnight. Treatment was successful in both patients achieving uncorrected vision of $6 / 6$ or better monocularly under high (100 per cent) and low (10 per cent) contrast conditions.

Results: At the six-month visit both patients presented with pigmented rings under slitlamp examination in both eyes. The location of the ring was consistent with the corneal area being flattened for myopic correction. Clinical examination was otherwise normal.

Conclusions: Despite being initially reported in Asian patients from Hong Kong, a pigmented ring related to orthokeratology treatment is also present in Caucasian patients, reducing the potential role of an ethnic link. Further large-scale studies need to be done to estimate the actual incidence of this condition and the potential implications for corneal health.

Key words: Caucasian, orthokeratology, pigmented ring, treatment zone

Orthokeratology has reinforced its role as a viable option to correct low-to-moderate myopia during the last 10 years. ${ }^{1}$ Current evidence that orthokeratology might slow down myopic progression has been found in at least four different studies and has increased the interest in this technique. ${ }^{2-5}$ The fitting procedure is predictable,${ }^{6}$ making thousands of patients independent of visual aids during the daytime. This expansion of the application of reverse geometry contact lenses to flatten the cornea overnight has allowed us to gain a better understanding of the histological and anatomical mechanisms of the procedure. A few complications have been associated with orthokeratology, including microbial keratitis, largely reported since $2005 .{ }^{7}$ In contrast, other corneal changes considered clinically irrelevant and reversible, such as a pigmented ring, ${ }^{8-10}$ fibrillary lines, ${ }^{11,12}$ white lesions $^{13}$ and other minor changes ${ }^{5,14,15}$ have been reported.
The advent of a pigmented ring has attracted interest from the clinical community because this has been reported in a significant number of Asian patients from Hong Kong ${ }^{9}$ and Taiwan. ${ }^{8}$ This feature is similar to the Fleischer ring observed quite often in patients with keratoconus $^{16}$ and similar features have been reported after laser-assisted in situ keratomileusis (LASIK) surgery. ${ }^{17}$

This article reports the observation of a pigmented ring in young adult Caucasian 


\begin{tabular}{|c|c|c|c|c|}
\hline & \multicolumn{2}{|c|}{ Patient 1} & \multicolumn{2}{|c|}{ Patient 2} \\
\hline Gender & \multicolumn{2}{|c|}{ Male } & \multicolumn{2}{|c|}{ Female } \\
\hline Age & \multicolumn{2}{|c|}{30} & \multicolumn{2}{|c|}{26} \\
\hline \multirow[t]{2}{*}{ Baseline manifest Rx } & \multicolumn{2}{|c|}{ RE: $-2.50 /-0.25 \times 170^{\circ}$} & \multicolumn{2}{|c|}{ RE: $-2.00 /-0.50 \times 165^{\circ}$} \\
\hline & \multicolumn{2}{|c|}{ LE: $-2.50 /-0.25 \times 165^{\circ}$} & \multicolumn{2}{|c|}{ LE: $-2.00 /-0.75 \times 165^{\circ}$} \\
\hline$S A_{100 \%}(\log M A R)$ & RE: -0.08 & LE: -0.06 & RE: -0.04 & LE: -0.02 \\
\hline $\mathrm{SVA}_{10 \%}(\log \mathrm{MAR})$ & RE: -0.02 & LE: -0.02 & RE: -0.00 & LE: 0.02 \\
\hline \multicolumn{5}{|l|}{ Baseline sim-Ks } \\
\hline Flattest meridian (D) & RE: 43.50 & LE: 43.75 & RE: 42.50 & LE: 42.75 \\
\hline Steepest meridian (D) & RE: 44.25 & LE: 44.50 & RE: 43.00 & LE: 43.25 \\
\hline \multicolumn{5}{|l|}{ Lens parameters } \\
\hline Base curve (mm) & RE: 8.40 & LE: 8.30 & RE: 8.50 & LE: 8.50 \\
\hline Return zone depth $(\mu \mathrm{m})$ & RE: 525 & LE: 525 & RE: 525 & LE: 525 \\
\hline Landing zone angle $\left({ }^{\circ}\right)$ & RE: 33 & LE: 33 & RE: 33 & LE: 32 \\
\hline \multicolumn{5}{|l|}{ Over-refraction } \\
\hline After treatment* & RE: +0.25 & LE: +0.50 & RE: -0.25 & LE: plano \\
\hline UCV $_{100 \%}(\log M A R)$ & RE: -0.02 & LE: -0.04 & RE: 0.00 & LE: -0.02 \\
\hline UCV $_{100 \%}(\log M A R)$ & RE: 0.10 & LE: 0.08 & RE: 0.12 & LE: 0.08 \\
\hline
\end{tabular}

Table 1. Patient data and lenses fitted

patients undergoing orthokeratology. Only a few cases of similar findings associated with orthokeratology have been published on non-Asian patients ${ }^{18}$ and this is the first report of the condition in Europe.

\section{CASE REPORTS}

Two Caucasian patients, one male and one female, were fitted with orthokeratology lenses to correct myopia between -1.75 and -2.50 DS with Paragon CRT (corneal refractive therapy) lenses. Treatment was successful in both patients, achieving uncorrected monocular vision of $6 / 6$ or better under high (100 per cent) and low (10 per cent) contrast conditions. At the six-month visit, both patients presented with a pigmented ring under slitlamp examination in both eyes. Visual acuity was recorded with the same high and low contrast retro-illuminated ETDRS (Early Treatment Diabetic Retinopathy Study) $\log$ MAR charts. Corneal topography was obtained with a Medmont E300 Corneal Topographer (Medmont Pty, Melbourne, Australia).

Patient 1 was a 30 -year-old man fitted with orthokeratology lenses for the first time. Spherical refractive error was $-2.50 \mathrm{D}$ in both eyes. The remaining data are provided in Table 1. Lenses fitted were Paragon CRT made of paflufocon D material $(\mathrm{Dk}=100$ barrer $)$ from Paragon Vision Science.

Figure 1 shows the fluorescein pattern with the lenses on the eye and the location of the pigmented ring seen under cobalt blue light. Figure 2 shows the tangential difference map of both eyes of patient 1 . The location of the ring was quite consistent with the corneal area being flattened with the purpose of myopic correction. Clinical examination appeared normal.

Patient 2 was a 26-year-old woman fitted with orthokeratology for the first time. Spherical refractive error was $-2.25 \mathrm{D}$ in the right eye and $-2.00 \mathrm{D}$ in the left. The remaining data are provided in Table 1. Lenses fitted were Paragon CRT made of paflufocon $\mathrm{D}$ material $(\mathrm{Dk}=100)$ from Paragon Vision Science.

Figure 3 shows the fluorescein pattern with the lenses on the eye and the location of the pigmented ring seen under cobalt blue light. Figure 4 shows the tangential difference map of both eyes of patient 2. Again, the location of the ring was apparently consistent with the corneal area being flattened with the purpose of myopic correction. Both patients were successful with the treatment for at least 12 months. Clinical examination appeared normal. Although this is an interesting finding, it does not seem to affect visual acuity, nor does it seem to be adverse in nature. Both patients were informed of their condition and neither chose to discontinue their overnight orthokeratology treatment.

\section{DISCUSSION}

A pigmented ring surrounding the treatment zone has been described as a relatively common condition in Asian patients undergoing overnight orthokeratology treatment; however, the condition had not been reported in non-Asian eyes, despite the spread of the treatment in countries such as The Netherlands, Spain and the United States of America. Indeed, there are very few reports in peer-reviewed literature about this condition. Cho and collegues $^{10}$ and Cho, Chui and Cheung ${ }^{19}$ found that the condition was reversible after treatment interruption and also found a direct link between the treatment zone of the lens and the location of the ring. Interestingly, they found a reduction in the diameter of the ring when they reduced the optic zone/treatment zone diameter of the lens. This geographical relationship seems to be true in the cases reported here, which suggests that there might be a direct relationship between the area treated and the appearance of the ring. Assuming that the changes occurring during orthokeratology are essentially limited to the epithelium, ${ }^{20,21}$ the pigmented ring might be a reflection of the 

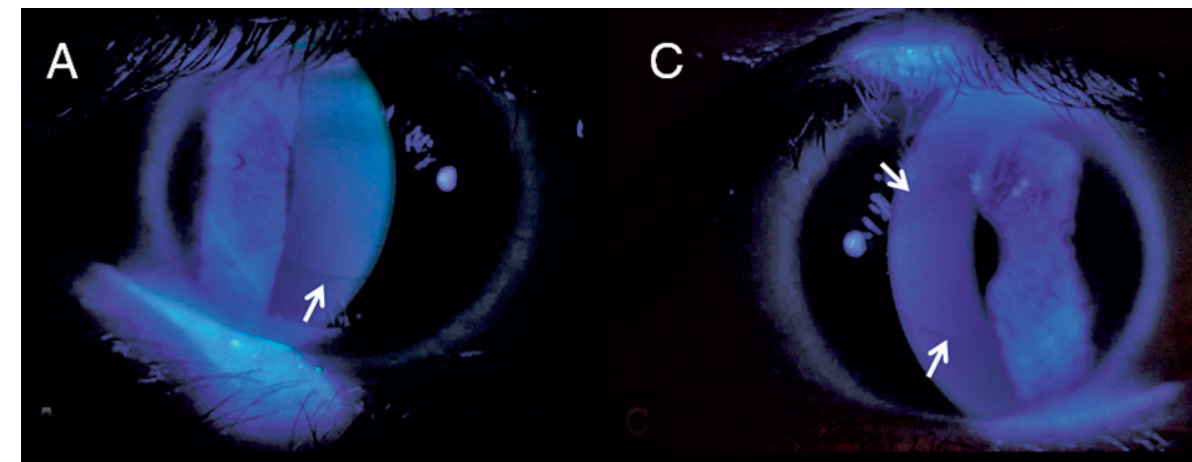

B

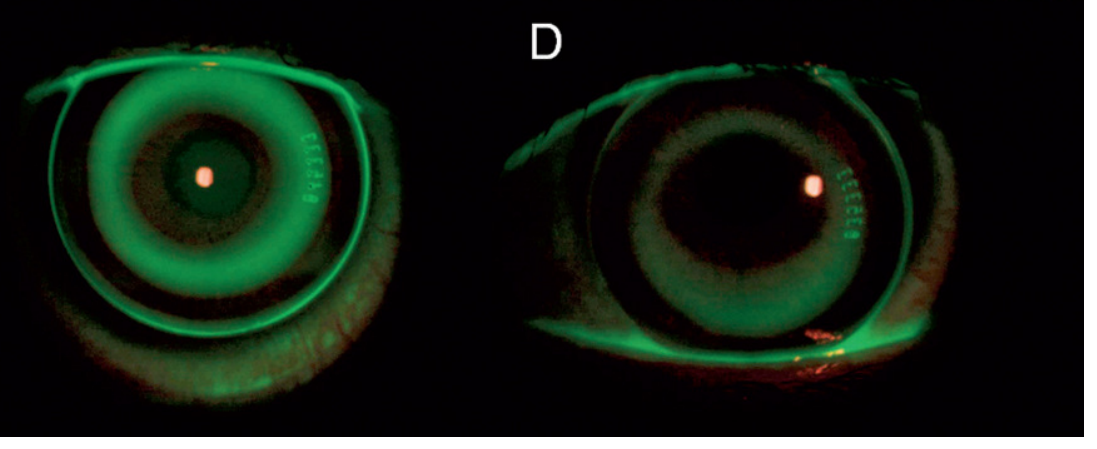

Figure 1. Appearance of pigmented ring under cobalt blue light and lens fitting showing fluorescein pattern for the right $(A, C)$ and left $(B, D)$ eyes of patient 1 . White arrows highlight the location of the pigmented ring.
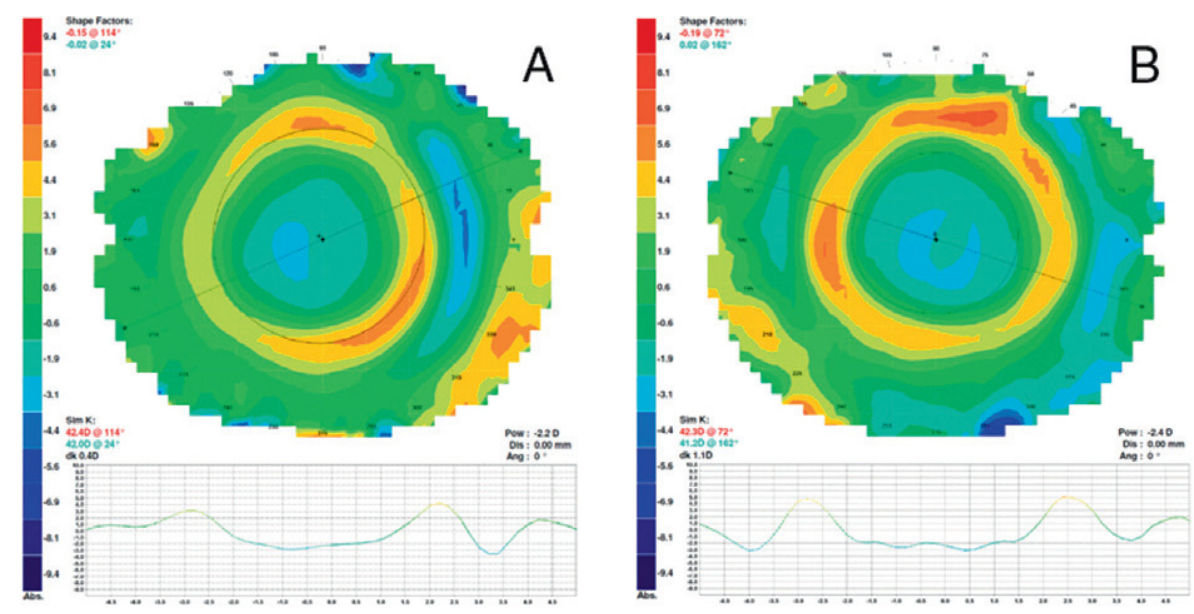

Figure 2. Corneal topography expressed as the tangential difference map for right (A) and left $(B)$ eyes of patient 1 stress forces applied to the epithelium or even tear stagnation in the reverse curve zone. ${ }^{9}$ Orthokeratology results in redistribution of the corneal epithelium, the central corneal epithelium thins and the mid-peripheral corneal epithelium thickens. ${ }^{20,22,23}$ The increased thickness of the epithelium in this area coupled with the decreased rate of desquamation seen in orthokeratology patients ${ }^{24}$ may provide for tear and cellular stasis, which most likely causes an increased rate of basal retention and stress forces may cause more prominent cellular and extracellular matrix degradation. In turn, this higher rate may result in more pigmented aggregation in the mid-peripheral thickened area of the epithelium. Following this line of reasoning, there might be a parallel between the aetiology of the Fleischer ring occurring in keratoconic corneas, which is assumed to have its origin in the epithelial basal layer. Both conditions are similar in appearance but a direct link concerning aetiology cannot be established solely based on this observation and the limited cases being reported.

Pigmented corneal deposits in a ring or patch have also been observed following LASIK surgery. ${ }^{17,25}$ This pigmented region within the margin of the ablated zone may offer insights into the dynamics of epithelial cell hyperplasia, as well as basal cell proliferation, differentiation and migration after LASIK.

Consistent with other pigment lines, the pigmentation is not near the visual axis and does not alter visual acuity. Therefore, no treatment is necessary. It is neither sight-threatening nor clinically significant. Considering the potential of orthokeratology to slow myopic progression in children $^{2-4}$ and the long-term wearing associated with this treatment, the aetiology of this condition and the potential implications in the long-term must be further evaluated.

One limitation of this report is the few cases reported. These patients come from a cohort of 28 patients being fitted for a short-term study, some of whom continued wearing the lenses for six months and beyond. As final year university students, most of those continuing to use the lenses 


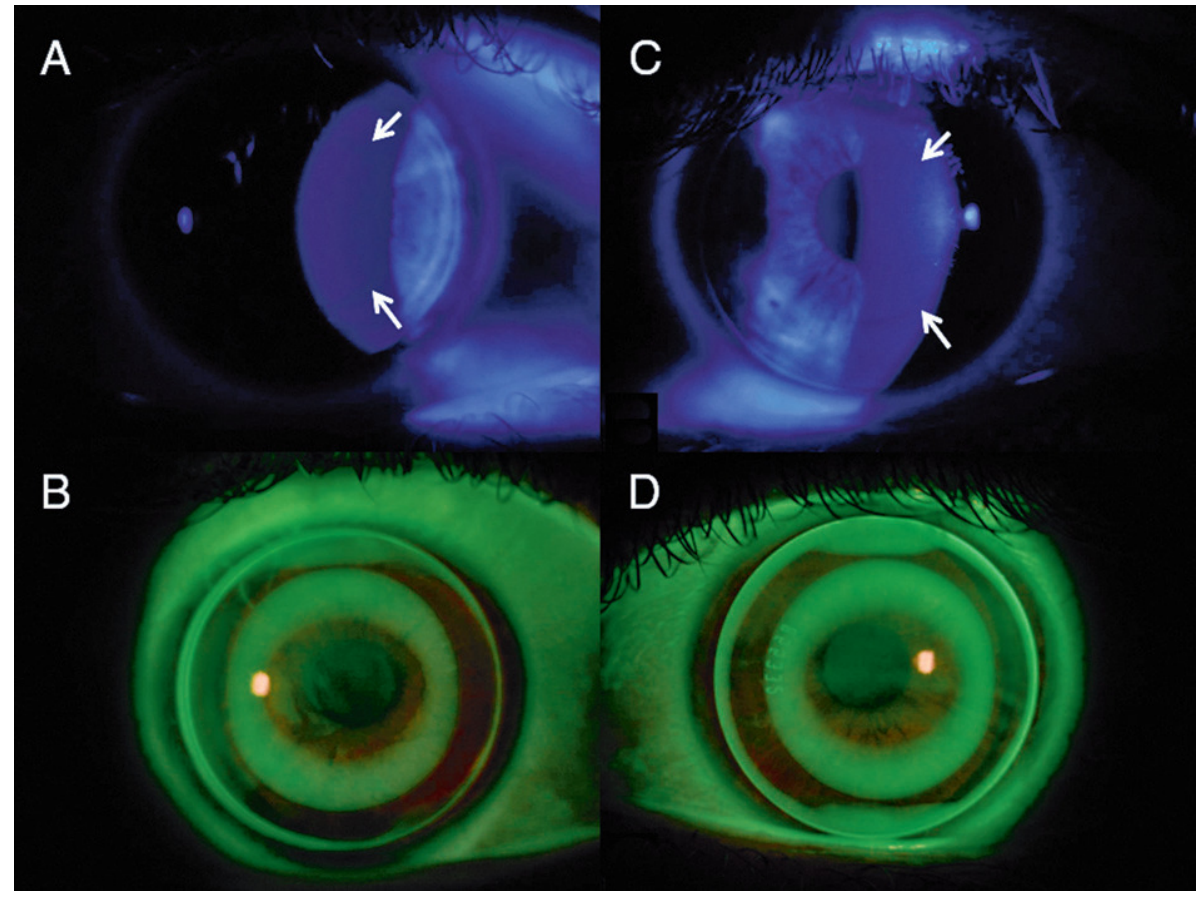

Figure 3. Appearance of pigmented ring under cobalt blue light and lens fitting showing fluorescein pattern for the right $(A, C)$ and left $(B, D)$ eyes of patient 2 . White arrows highlight the location of the pigmented ring.
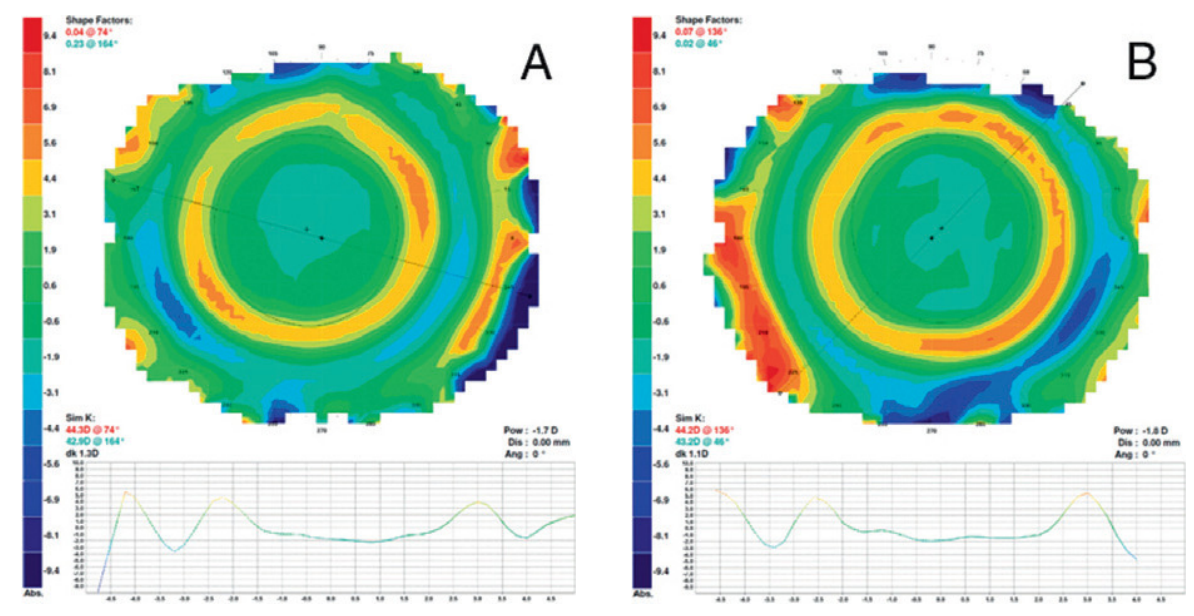

Figure 4. Corneal topography expressed as the tangential difference map for right (A) and left (B) eyes of patient 2 did their follow-up outside the Clinical and Experimental Optometry Research Laboratory. Of the 10 being followed up to the sixth-month visit, only the two reported here presented with a clinically evident pigmented ring. We cannot ensure that other patients of the same ethnicity will not have this condition and this deserves a closer observation during future visits by our clinical population. Although is not possible to provide a value for the incidence of this condition in nonAsian eyes from the present series, unpublished data from our group indicate that corneal iron deposits were present in 70 per cent of cases of overnight orthokeratology lens wear in Spain. Cho and colleagues ${ }^{10}$ reported an incidence of 90 per cent after 12 months in Hong Kong. Vongthongsri, Chuck and Pepose ${ }^{25}$ reported this finding in 42 per cent of LASIK patients. Furthermore, both studies found a positive correlation between the amount of pre-treatment myopia and the incidence of pigmented ring. In both patients reported here, the baseline refractive errors were relatively small. A similar ring formation (Fleischer's ring) has been found in 86 per cent of keratoconus patients in the CLEK study by Zadknik and colleagues $^{26}$. Thus, there might be substantial variability in the incidence of a pigmented ring in patients with keratoconus or undergoing orthokeratology that can be affected by the examination conditions, but also to the intrinsic characteristics of the eye, such as iris colour, ${ }^{27}$ that constitutes the background against which the ring is observed. In the present report, both patients had brown irises that might help to identify the presence of the pigmented ring.

A prospective clinical study should examine the occurrence of this condition in the general population undergoing orthokeratology. Correlations with baseline corneal curvature, corneal thickness and the target refractive correction might be analysed, looking for predictive variables for the manifestation of this condition. The potential involvement of the biomechanical rigidity of the cornea on the appearance of this condition will also be of interest. ${ }^{28,29}$ 
New technological advances, such as confocal microscopy or spectral domain optical coherence tomography ${ }^{30}$ may shed light on the extent and location of this condition in the cornea and elucidate its aetiology and eventual clinical significance. Molecular studies are being done by our research group to elucidate potential correlates with its appearance.

\section{REFERENCES}

1. Villa-Collar C, González-Méijome JM. Ortoqueratologia Nocturna. Madrid: ICM-Colegio Nacional de Ópticos-Optometristas de España, 2006.

2. Cho P, Cheung SW, Edwards M. The longitudinal orthokeratology research in children (LORIC) in Hong Kong: a pilot study on refractive changes and myopic control. Curr Eye Res 2005; 30: 71-80.

3. Walline JJ, Jones LA, Sinnott LT. Corneal reshaping and myopia progression. Br J Ophthalmol 2009; 93: 1181-1185.

4. Kakita T, Hiraoka T, Oshika T. Influence of overnight orthokeratology on axial elongation in childhood myopia. Invest Ophthalmol Vis Sci 2011; 52: 2170-2174.

5. Santodomingo-Rubido J, Villa-Collar C, Gilmartin B, Gutiérrez-Ortega R. Myopia control with orthokeratology contact lenses in Spain (MCOS): Study design and general baseline characteristics. $J$ Optom 2009; 2: 215-222.

6. González-Méijome JM, Villa-Collar C. Nomogram, corneal topography, and final prescription relations for corneal refractive therapy. Optom Vis Sci 2007; 84: 59-64.

7. Watt KG, Swarbrick HA. Trends in microbial keratitis associated with orthokeratology. Eye Contact Lens 2007; 3: 373-377.

8. Liang JB, Chou PI, Wu R, Lee YM. Corneal iron ring associated with orthokeratology. J Cataract Refract Surg 2003; 29: 624-626.

9. Cho P, Chui WS, Mountford J, Cheung SW. Corneal iron ring associated with orthokeratology lens wear. Optom Vis Sci 2002; 79: 565-568.

10. Cho P, Cheung SW, Mountford J, Chui WS. Incidence of corneal pigmented arc and factors associated with its appearance in orthokeratology. Ophthalmic Physiol Opt 2005; 25: 478-84.

11. Cheung SW, Cho P, Bron AJ, Chui V, Chan B. Case report: the occurrence of fibrillary lines in overnight orthokeratology. Ophthalmic Physiol Opt 2006; 26: 525-531

12. Lum E, Swarbrick H. Fibrillary lines in overnight orthokeratology. Clin Exp Optom 2007; 90: 299302.

13. Cheung SW, Cho P, Cheung A. White lesion in the corneal pigmented ring associated with orthokeratology. Ophthalmic Physiol Opt 2005; 25: 264-268.

14. Nichols JJ, Marsich MM, Nguyen M, Barr JT, Bullimore MA. Overnight orthokeratology. Optom Vis Sci 2000; 77: 252-259.

15. Walline JJ, Rah MJ, Jones LA. The Children's Overnight Orthokeratology Investigation (COOKI) pilot study. Optom Vis Sci 2004; 81: 407413.

16. Hiratsuka Y, Nakayasu K, Kanai A. Secondary keratoconus with corneal epithelial iron ring similar to Fleischer's ring. Jpn J Ophthalmol 2000; 44: 381386.
17. Probst LE, Almasswary MA, Bell J. PseudoFleischer ring after hyperopic laser in situ keratomileusis. J Cataract Refract Surg 1999; 25: 868-870.

18. Rah MJ, Barr JT, Bailey MD. Corneal pigmentation in overnight orthokeratology: a case series. Optometry 2002; 73: 425-434.

19. Cho P, Chui WS, Cheung SW. Reversibility of corneal pigmented arc associated with orthokeratology. Optom Vis Sci 2003; 80: 791-795.

20. Alharbi A, Swarbrick HA. The effects of overnight orthokeratology lens wear on corneal thickness. Invest Ophthalmol Vis Sci 2003; 44: 2518-2523.

21. Haque S, Fonn D, Simpson T, Jones L. Corneal and epithelial thickness changes after 4 weeks of overnight corneal refractive therapy lens wear, measured with optical coherence tomography. Eye Contact Lens 2004; 30: 189-193.

22. Swarbrick HA, Wong G, O'Leary DJ. Corneal response to orthokeratology. Optom Vis Sci 1998; 75: 791-799.

23. Lu F, Simpson T, Sorbara L, Fonn D. Corneal refractive therapy with different lens materials, part 2: effect of oxygen transmissibility on corneal shape and optical characteristics. Optom Vis Sci 2007; 84: 349-356.

24. Guo Y, Nguyen T, Soni S. Cell shedding in overnight orthokeratology. Invest Ophthalmol Vis Sci 2004; 45: 1581.

25. Vongthongsri A, Chuck RS, Pepose JS. Corneal iron deposits after laser in situ keratomileusis. $A m$ J Ophthalmol 1999; 127: 85-86.

26. Zadnik K, Barr JT, Edrington TB, Everett DF, Jameson M, McMahon TT, Shin JA et al. Baseline findings in the Collaborative Longitudinal Evaluation of Keratoconus (CLEK) Study. Invest Ophthalmol Vis Sci 1998; 39: 2537-2546.

27. Franssen L, Joris EC, van den Berg TJTP. Grading of iris color with an extended photographic reference set. J Optom 2008; 1: 36-40.

28. González-Méijome JM, Villa-Collar C, Queirós A, Jorge J, Parafita MA. Pilot study on the influence of corneal biomechanical properties over the short term in response to corneal refractive therapy for myopia. Cornea 2008; 27: 421-426.

29. Chen D, Lam AK, Cho P. A pilot study on the corneal biomechanical changes in short-term orthokeratology. Ophthalmic Physiol Opt 2009; 29: 464-471.

30. González-Méijome JM, Cerviño A, Carracedo G, Queirós A, Garcia-Lazaro S, Ferrer-Blasco T. Highresolution spectral domain optical coherence tomography technology for the visualization of contact lens to cornea relationships. Cornea 2010; 29: 1359-1367.

Corresponding author:

Dr Jose Manuel Gonzalez-Meijome

CEORLab-Center of Physics

\section{School of Science}

University of Minho

Gualtar

4710-057 Braga

PORTUGAL

E-mail: jgmeijome@fisica.uminho.pt 